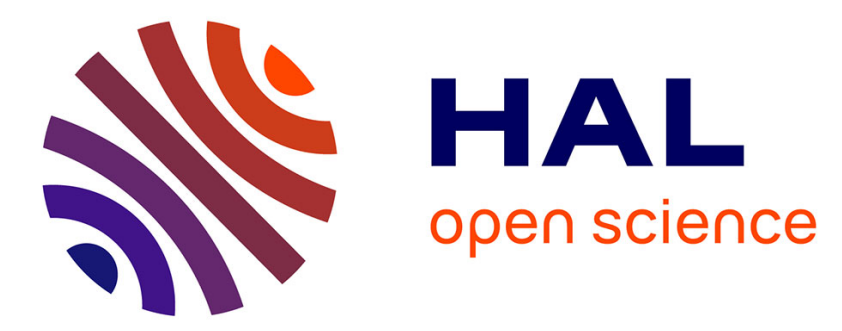

\title{
Low-frequency behavior of laminated electric steel sheet: investigation of ferromagnetic hysteresis loops and incremental permeability
}

\author{
S. Zhang, Benjamin Ducharne, S. Takeda, G. Sebald, Tetsuya Uchimoto
}

\section{- To cite this version:}

S. Zhang, Benjamin Ducharne, S. Takeda, G. Sebald, Tetsuya Uchimoto. Low-frequency behavior of laminated electric steel sheet: investigation of ferromagnetic hysteresis loops and incremental permeability. Journal of Magnetism and Magnetic Materials, In press, pp.168278. 10.1016/j.jmmm.2021.168278 . hal-03283606

\section{HAL Id: hal-03283606 https://hal.science/hal-03283606}

Submitted on 12 Jul 2021

HAL is a multi-disciplinary open access archive for the deposit and dissemination of scientific research documents, whether they are published or not. The documents may come from teaching and research institutions in France or abroad, or from public or private research centers.
L'archive ouverte pluridisciplinaire HAL, est destinée au dépôt et à la diffusion de documents scientifiques de niveau recherche, publiés ou non, émanant des établissements d'enseignement et de recherche français ou étrangers, des laboratoires publics ou privés. 


\title{
Low-frequency behavior of laminated electric steel sheet: investigation of ferromagnetic hysteresis loops and incremental permeability
}

\author{
S. Zhang ${ }^{1,2}$, B. Ducharne ${ }^{3,4}$, S. Takeda ${ }^{2}$, G. Sebald ${ }^{3}$, T. Uchimoto ${ }^{2}$
}

1. Graduate School of Engineering, Tohoku University, Sendai, Japan

2. Tohoku University, Institute of fluid science IFS, Sendai, Japan.

3. ELyTMaX UMI 3757, CNRS - Université de Lyon - Tohoku University, International Joint Unit, Tohoku University, Sendai, Japan.

4. LGEF, INSA Lyon, Villeurbanne, France. 


\begin{abstract}
:
The frequency dependence of ferromagnetic hysteresis and the related core losses is still an open question. From scaling relations up to space discretized and simultaneous contributions resolutions, different levels of accuracy in the estimation of these losses can be reached. Below a given frequency known as the quasi-static threshold, it is well admitted that the hysteresis cycle remains unchanged. A precise evaluation of this threshold is fundamental to simulate and evaluate precisely the magnetization behaviors under higher frequencies. But this estimation is complex, especially through the unique observation of the hysteresis cycles and because of instrumentation limitations. In this manuscript, we propose an alternative and simple method to precisely refine this threshold's estimation. By using an alternative magnetic signature known as the magnetic incremental permeability butterfly loop $\left(\left|\underline{\mu_{I P}}\right|(H)\right)$, improvements are proposed in observing the very low-frequency magnetization behaviors and assessing the quasi-static threshold. This work constitutes a leap forward in understanding the magnetic low-frequency behaviors of laminated electric steel sheets.
\end{abstract}

\title{
Keywords :
}

Quasi-static behavior, magnetic incremental permeability, ferromagnetic hysteresis, frequency dependence. 


\section{1 - Introduction}

Ferromagnetic laminated cores can be found in many electromagnetic devices used to convert energy through a magnetic way. The electromagnetic conversion efficiency has been continuously improved, fueled by constant signs of progress in understanding ferromagnetic materials. Generations of researchers have dedicated their works to this problem, but questions remain open. The dynamic dependence of the magnetization processes is one of them. Even if the first manuscript on this topic has been published in the middle of the $20^{\text {th }}$ century [1][2], the number of recent (last year) publications is impressive [3]-[11]. A plausible explanation for this surge of interest can be found in the evolution of electromagnetic devices: faster, compacter, and more efficient, forcing the magnetic materials over unprecedented dynamic conditions.

The frequency dependence of the ferromagnetic laminated cores has multiple origins: some of them, like the domain wall bulging, the domain wall displacement, and the magnetization rotation, take place at the micrometric scale. Others, like the magnetic field diffusion and the related macroscopic eddy currents, occur at a much larger scale. Each of these behaviors is characterized by its own time constant and its own frequency dependence [12]. They all manifest themselves through the dynamic evolution of the hysteresis cycle. The hysteresis cycle is the standard magnetic signature. It gives the variations of the magnetic induction $B_{a}$ (averaged through the cross-section) as a function of the tangent surface magnetic excitation $H_{\text {surf }}$, assuming both these vector quantities in a collinear situation. Below a given frequency known as the quasi-static threshold, the frequency dependence of the hysteresis cycle becomes insignificant, and the hysteresis shape remains unchanged. According to the well-admitted Statistical Theory of Losses (STL) [13]-[15], beyond the quasi-static threshold (in the upward 
direction) under sinusoidal $B_{a}$ imposed conditions, the hysteresis area of a laminated electric steel sheet (also known as the magnetic losses $W$ ) follows the trajectory depicted in Fig. 1 below:

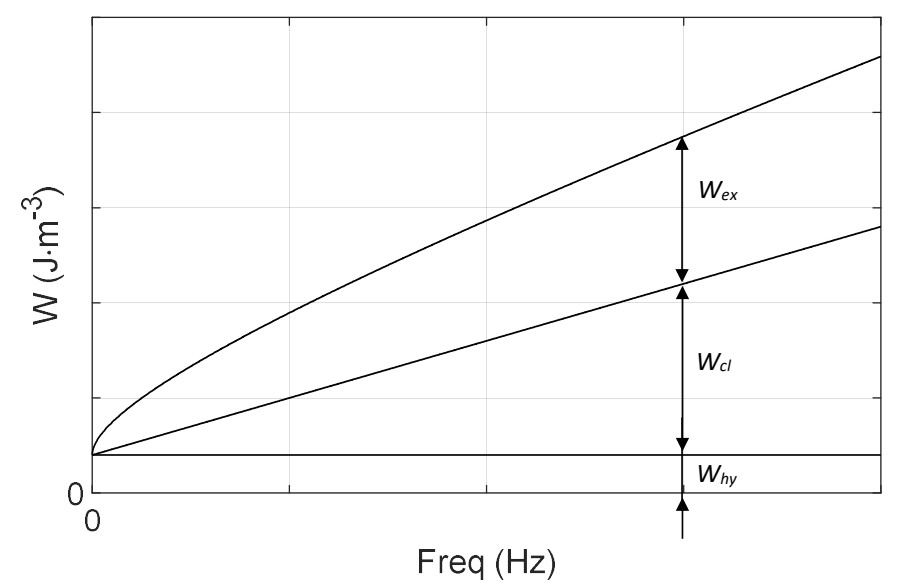

Fig. 1 - Magnetic losses $W$ distribution as a function of the frequency.

In [14], Bertotti proposed the following mathematical expression for the simulation of these magnetic losses:

$$
\begin{gathered}
W=W_{h y}+W_{c l}+W_{e x} \\
W_{c l}=\frac{\sigma d^{2}}{12} \int_{0}^{1 / f}\left(\frac{d B_{a}}{d t}\right)^{2} d t \\
W_{e x}=\sqrt{\sigma S G V_{0}} \int_{0}^{1 / f}\left|\frac{d B_{a}}{d t}\right|^{1.5} d t
\end{gathered}
$$

Here, $W_{h y}, W_{c l}, W_{e x}$ are the hysteresis losses, the classic losses, and the excess losses, respectively. $G=0.1356$ is a dimensionless coefficient, $V_{0}$ a statistical parameter linked to the microstructure, $d$ the lamination thickness, and $\sigma$ the electrical conductivity. STL is remarkable in many ways, including its applicability to various materials (electrical steel, Armco iron, NiFe alloys ...). Still, STL also shows limitations mostly due to the ignorance of the skin effect, which restricts the efficiency of the method to the low-frequency range ( < to a few hundred hertz in the case of typical electrical steels) [16]. In [17][18], alternative developments were proposed to 
improve the magnetic hysteresis frequency dependence simulation. In both cases, the magnetic diffusion equation (Eq. 4) was solved using a 1D finite differences method and replacing the vector quantities by their magnitudes, assuming local $B$ and $H$ in a colinear situation.

$$
\nabla^{2} \vec{H}=\sigma \frac{d \vec{B}}{d t} \rightarrow \frac{\partial^{2} H}{\partial z^{2}}=\sigma \frac{d B}{d t}
$$

Where $z$ is the depth direction. Interesting development can be found in [19] for the establishment and resolution of Eq. 4, including the expression of the boundary and initial conditions. The resolution of Eq. 4 in itself is impossible and only goes through the simultaneous resolution of a so-called material law. The material law bonds the discretized $B_{i}$ and $H_{i}$ locally. Its simplest expression is given below:

$$
\begin{gathered}
B_{i}=\mu_{0} \mu_{r} H_{i} \\
B_{a}=\frac{\sum B_{i}}{n}
\end{gathered}
$$

Where $n$ is the space discretization number of nodes. Solving Eq. 4 and 5 simultaneously is relatively straightforward, but the resulting magnetic losses are limited to the classic losses (Eq. 1). Bonding $B_{i}$ and $H_{i}$ using a frequency-independent hysteresis model (Eq. 7) like the J-A model or the Preisach model [20][21] in their original configuration is another option. It improves the magnetic losses $W$ simulation by adding the hysteresis losses $W_{h y}$ taking into account but the excess losses term $W_{\text {ex }}$ (Eq. 1) is still missing.

$$
B_{i}=f_{\text {static }}\left(H_{i}\right)
$$

Due to hysteresis, $f_{\text {static }}$ cannot be expressed analytically. It connects the input and the output of the quasi-static hysteresis model for every simulation step. 
A complete consideration of $W$ can eventually be obtained by giving a frequency dependence of the material law. Different options have already been tested for the frequency dependence of this material law [22], eq. 8 below is one of them:

$$
\rho\left(\frac{d B_{i}}{d t}\right)=H_{i}-f_{\text {static }}^{-1}\left(B_{i}\right)
$$

but excellent results have been obtained with Eq. 9:

$$
\delta\left|\beta\left(\frac{d B_{i}}{d t}\right)\right|^{0.5}=H_{i}-f_{\text {static }}^{-1}\left(B_{i}\right)
$$

Where $\rho, \beta$ can be constant and defined as specimen parameters or expressed as a function of $B$ for an even better accuracy [18][23]. $\delta= \pm 1$ a directional variable [18]. Fig. 2 below gives the low-frequency dependence of $W$ (Fig. 2 - b), and of the coercivity $H_{c}$ (Fig. 2 - c) as obtained using Eq. 8 for the material law. For these simulations, $B_{a}$ was imposed sinus with a maximum value equal to $1.4 \mathrm{~T}$, and the inverse Jiles-Atherton (J.A. ${ }^{-1}$ ) model [24] was used for the $f_{\text {static }}{ }^{-1}\left(B_{i}\right)$ contribution. The simulation parameters are given at the top right-hand side, and some of the related hysteresis cycles are shown on the left-hand side. For these parameters, we used those of a typical Non-Oriented (NO) grains electric steel lamination collected in [25]: 


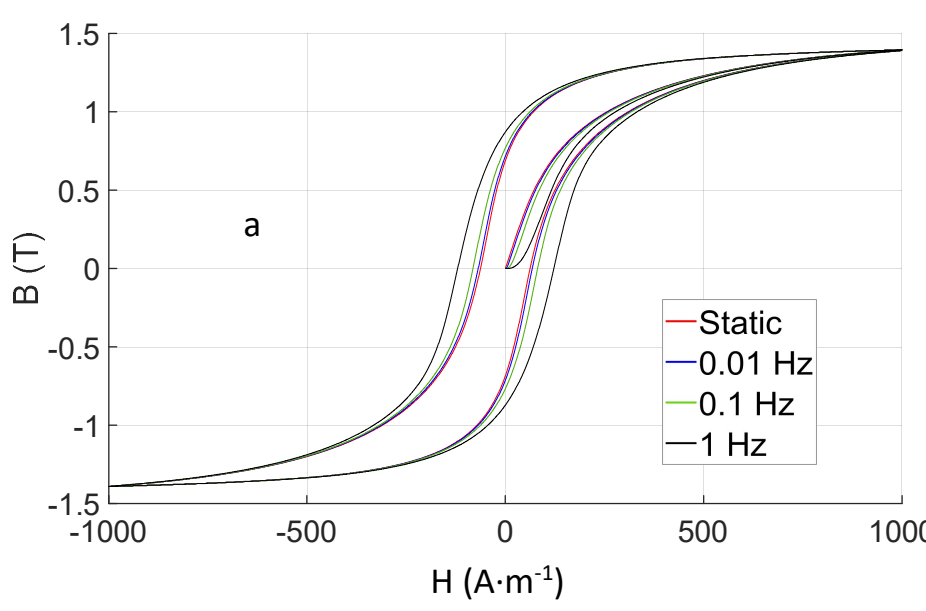

\begin{tabular}{c|c} 
J-A - $^{-1}$ parameters & Typical value \\
$\alpha$ & 0.00017239 \\
$\mathrm{k}\left(\mathrm{A} \cdot \mathrm{m}^{-1}\right)$ & 285.3 \\
$\mathrm{c}$ & 0.6397 \\
$\mathrm{a}\left(\mathrm{A} \cdot \mathrm{m}^{-1}\right)$ & 90.8512 \\
$\mathrm{Ms}\left(\mathrm{A} \cdot \mathrm{m}^{-1}\right)$ & 1226138 \\
& \\
Dynamic parameters & Typical value \\
\hline$\left(\mathrm{A} \cdot \mathrm{m}^{-1} \cdot \mathrm{s} \cdot \mathrm{T}^{-1}\right)$ & 50 \\
$\sigma\left(\mathrm{S} \cdot \mathrm{m}^{-1}\right)$ & 2000000 \\
Space step dx $(\mathrm{m})$ & 0.0000175 \\
Specimen thickness $(\mathrm{m})$ & 0.00035 \\
&
\end{tabular}
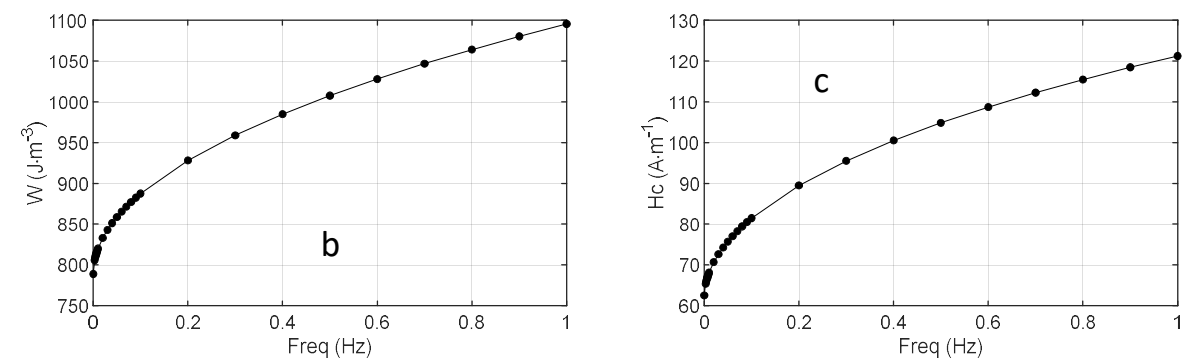

Fig. 2 - a Low-frequency $B(H)$ simulated hysteresis cycles, Fig. 2 - b Hysteresis area variations as a function of the frequency $W(f)$, Fig. 2 - c Coercivity variations as a function of the frequency $H_{c}(f)$.

If we specifically focused on the low-frequency behavior, it is worth noting the frequency effect affecting similarly $H_{c}$, and $W$. This result is evident on the $H_{c}(W)$ curve (Fig. 3) below:

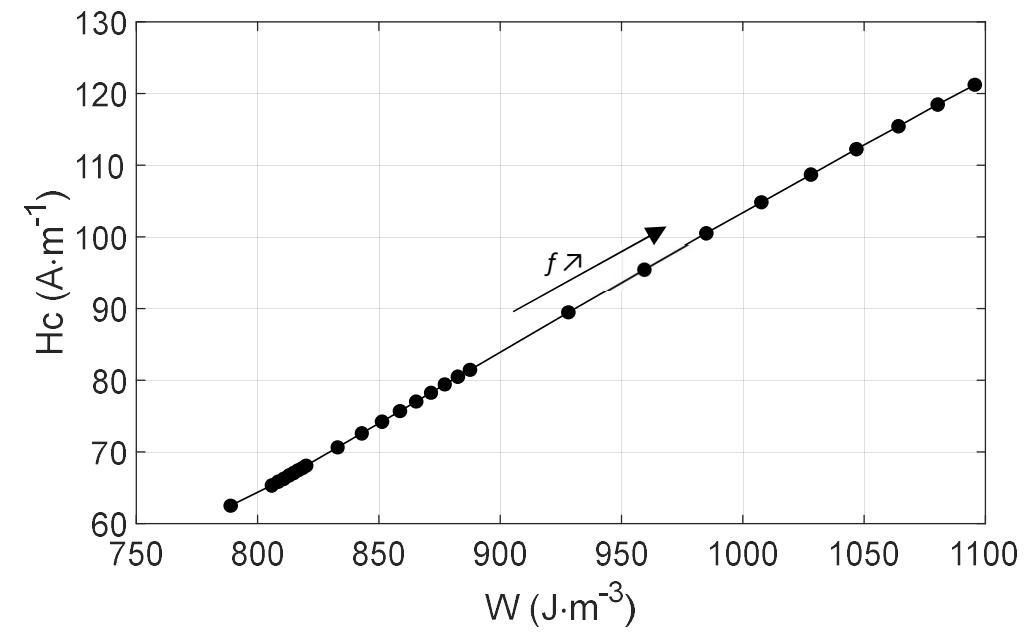

Fig. 3 - Coercivity variations as a function of the hysteresis $H c(<A>)$ under frequency increasing situation. 
The correlation coefficient between $H_{c}$, and $W$ calculated with Matlab ${ }^{\circledR}$ "corr" function is equal to 0.9999 , confirming that in the low-frequency range, both indicators can be equally used to evaluate the frequency effect correctly.

Recently, alternative approaches using operators from the framework of the fractional derivative have been proposed for the simulation of the ferromagnetic hysteresis frequency dependence [25]-[29]. Anomalous fractional diffusion or fractional differential equations can be solved separately or associated with the classic methods to reach accurate results on extremely large frequency bandwidths. Time fractional derivative operators are intrinsically long memory dependent, especially well adapted to hysteresis. $n$ the fractional-order constitutes an additional degree of freedom providing flexibility in the simulation process and improving the accuracy.

To conclude with this general overview of the ferromagnetic hysteresis frequency dependence, we can confirm that accurate simulation tools already exist. Still, limitations remain, and discrepancies can be observed for the extreme frequency levels of the working conditions, very low $(<0.1 \mathrm{~Hz})$, and very high $(>1 \mathrm{kHz})$ frequency range. Multiple plausible reasons can be suggested to explain these limitations, including in the first line the lack of reliable experimental results.

In this manuscript, we especially focus on the very low dynamic dependence of the magnetization behaviors. According to the simulation results depicted in Fig. 2, variations are significant in the low-frequency range, and experimental validations specifically complex to obtain. Classic experimental set-ups for the electric steel magnetic characterization as described by the IEC international standards (IEC 60404 - 1-3) always use B-coils wound around the tested specimen to observe the magnetic Induction $B$ or the magnetic polarisation $J$ [30]-[33]. By 
assuming the specimen magnetic state in a demagnetized configuration, a good approximation of $B(t)$ can be obtained through the resolution of Eq. 11 below, where $n$ is the B-coil number of turns, $\emptyset$ the magnetic flux and $S$ the specimen cross-section:

$$
\begin{gathered}
e(t)=n \frac{d \emptyset}{d t} \\
B(t)=\frac{1}{n \cdot S} \int_{0}^{t} e(t) d t \\
\mu_{D P}(t)=\frac{d B(t)}{d H(t)}=\frac{1}{n s \frac{d H(t)}{d t}} e(t)
\end{gathered}
$$

Eq. 12 above gives the time dependence of the Magnetic Differential Permeability (MDP, $\left.\left|\underline{\mu_{D P}}\right|\right)$. Just like the usual hysteresis cycles, plotted as a function of $H_{s u r f}$, it can be used as an indicator of the magnetization frequency dependence.

Eq. 10 gives the electromotive force due to the magnetic flux variations during the magnetization process. In the low-frequency range, extremely weak levels can be reached: $\approx 20$ $\mu \mathrm{V}$ for a 200 turns B-coil at $1 \mathrm{mHz}, 1.5 \mathrm{~T}$, and $30 \times 0.35 \mathrm{~mm}$ cross-section. Such a weak amplitude is complex to manipulate, and a precise estimation of the related hysteresis cycle, magnetic loss, and dynamic dependence almost impossible. In most cases, the weak dynamical contribution under low-frequency levels is ignored, and the quasi-static threshold is set in an overestimated frequency.

In this manuscript, we proposed to refine the low-frequency dependence of the magnetization behaviors by observing them differently. The Magnetic Incremental Permeability (MIP, $\left|\underline{\mu_{I P}}\right|$ ) (defined in subsection 2-a) and more specifically, the MIP butterfly loops $\left|\underline{\mu_{I P}}\right|\left(H_{\text {surf }}\right)$ of NO electric steel specimens are measured at different levels of low-frequency excitation field (from $1 \mathrm{mHz}$ to $1 \mathrm{~Hz}$ ). These butterfly loops are compared to the real static one 
obtained under the constant excitation field of amplitude changed manually. A parallel between the $\mu_{I P}$ and the $\mu_{D P}$ frequency dependences is established by comparing them in a frequency window where both these signatures can be measured precisely (from $100 \mathrm{mHz}$ to $1 \mathrm{~Hz}$ ). The very similar variations of $H_{c_{\mu I P}}(f)$ and $H_{c_{\mu D P}}(f)$ frequency dependence of both the coercivities allows us to conclude that the dynamic of the MIP curve follows the same trajectory as the $B_{a}\left(H_{\text {surf }}\right)$ one. Based on this notation, the low-frequency dynamics of the tested specimens are estimated precisely by the observation of the accurate $\left|\underline{\mu_{I P}}\right|(H)$ curves instead of the usual approximate $B_{a}\left(H_{\text {surf }}\right)$ hysteresis loops. Especially, an analytical expression of $H_{c_{\mu I P}}(f)$ is provided to address quantitatively the very low-frequency magnetization behavior. Then, a precise estimation of the quasi-static threshold is obtained by superimposing this curve to the real static value obtained with constant excitation field bias of amplitude changed manually.

This manuscript is organized as follows:

The first section is dedicated to the description of the magnetic incremental permeability, the experimental set-up, and the tested specimens. The second section to the experimental results and the last section to the discussion and conclusions.

\section{2 - Experimental set-up}

\section{2-a) Introduction to magnetic incremental permeability}

The superimposition of a slowly varying, high amplitude magnetic excitation field $H_{D C}$ to a low amplitude, high frequency ECT-type excitation $H_{A C}$ (pancacke coil impedance monitoring with an LCR meter, as illustrated in Fig. 4) enables the measurement of the MIP signature: the 
butterfly loop (evolution of the ECT coil impedance modulus $|\underline{Z}|$ or of the incremental permeability modulus $\left|\underline{\mu_{I P}}\right|$ as a function of the $H_{D C}$ magnetic field).

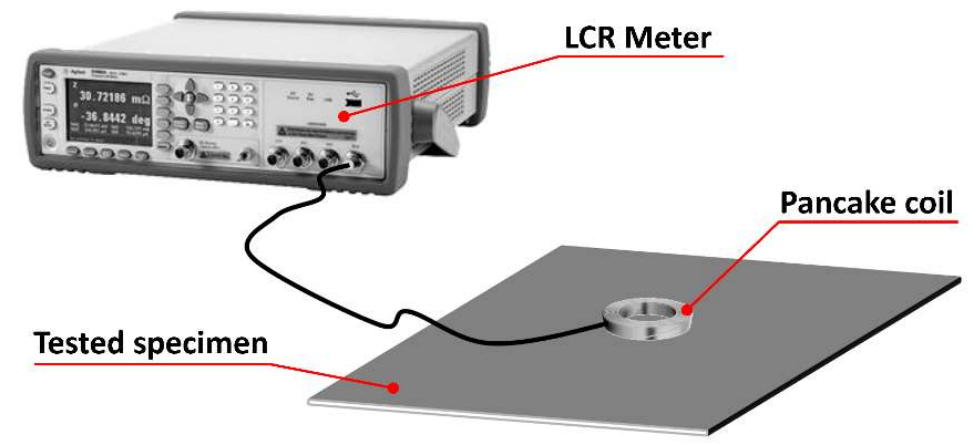

Fig. 4 - Eddy current nondestructive testing of a flat specimen.

The simultaneous magnetic excitation $H_{D C}+H_{A C}$ generates a major hysteresis cycle trajectory slightly augmented from a large quantity of minor low amplitude asymmetric loops. The raw measurement as provided by the impedance meter gives the complex impedance of the sensor coil $|\underline{Z}|$ but not the corresponding permeability. However, the well-known and accurate Dodd \& Deeds (D\&D) simulation method can be used to precisely convert the measured impedance into the corresponding impedance $|\underline{\mu}|$ [23][34]-[36]. This conversion is not direct and goes through the establishment of a template curve $\mu_{r}|Z|$ obtained by simulating the experimental situation considering the permeability as a parameter and testing it within a large window $\left(0<\mu_{r}<5000\right)$. Fig. 5 below depicted the $\mu_{r}|Z|$ template curve obtained for the sensor coil described in sub-section 2-b. 


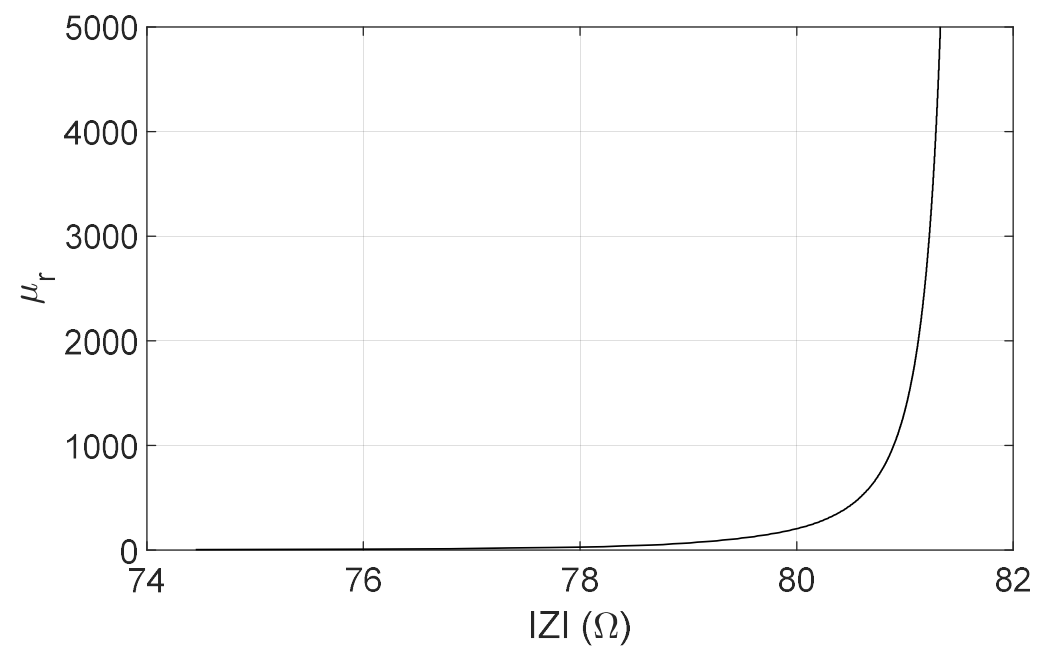

Fig. 5 - Relative permeability as a function of the impedance (template curve) obtained with the D\&D simulation ( $f$ $=50 \mathrm{kHz}$ )

As $\mu_{r}|Z|$ is strictly increasing, the relative permeability associated with a given impedance can be obtained by interpolation.

It is worth mentioning that improved accuracy of the MIP butterfly can be observed by replacing the ECT coil with the combination of a Transmitter/Receiver coils sensor and a lock-in amplifier [37].

MIP has received considerable attention in the NDT\&E services of the steel manufacturers and steel end-users industry as it is a remarkable indicator of mechanical residual stresses and strain [38][39]. MIP gives stable indicators well adapted to evaluate the mechanical sub-surface properties of tested specimens and their evolutions (creep, structural health monitoring ...). Finally, MIP can be used on flat and oversized specimens as surrounding coils are not required.

\section{2-b) Experimental set-ups}

Extremely low-frequency observation of the magnetization behaviors is complex due to the weak amplitude of the induced electromotive forces. There is an obvious parallel (as verified and 
described in the third section of this manuscript) between the frequency dependence of $\mu_{D P}, \mu_{I P}$ and of the hysteresis cycle $B_{a}\left(H_{\text {surf }}\right)$, but the low-frequency behavior is easier to observe through the MIP butterfly signature $\left|\underline{\mu_{I P}}\right|\left(H_{\text {surf }}\right)$.

The first step of this research experimental tests consisted in the simultaneous acquisition of $B_{a}\left(H_{\text {surf }}\right)$ and of the MIP butterfly signature: evolution of the sensor coil impedance modulus $|\underline{Z}|$ or of the incremental permeability $\left|\underline{\mu_{I P}}\right|$ as a function of the tangent magnetic field excitation $H_{\text {surf }}$. A dedicated experimental set-up has been developed especially for this (Fig. $6-$ a).

A DC power amplifier (HSA 4014 by NF Corporation) driven by a frequency generator (Agilent 33220A) fed in low frequency current a 200 turns wound coil. This primary coil was associated with a U-shaped large section magnetic yoke for the generation of the $H_{D C}$ low-frequency magnetic field excitation contribution (see Fig. 6 below). A shunt resistor plugged in series to the wound coil was used to observe precisely the current $I$ and return the magnetic excitation $H_{\text {surf }}$ indirectly. Both these quantities are linked through the Ampère theorem (Eq. 13), where $L$ is supposed to be the mean magnetic path length:

$$
H_{\text {surf }} \cdot L=N \cdot I
$$

$B$ was measured with a 500 turns B-coil wound around the tested specimen. The integration step was done numerically. Both drift and magnetic leakages corrections were performed in postprocessing.

A pancake coil (Eddy Current Testing (ECT) coil) was placed on the surface of the specimen between the legs of the yoke. The dimensions of the ECT coil were as follows: $3.3 \mathrm{~mm}$ inner diameter, $3.95 \mathrm{~mm}$ outer diameter, $3.0 \mathrm{~mm}$ height, 275 turns, and $0.05 \mathrm{~mm}$ wire thickness. The lift-off was $0.39 \mathrm{~mm}$. The coil impedance was measured using an LCR meter (ZM2375, NF 
Corporation). All the LCR meter measurements are obtained with an error of less than $1 \%$. The current of the ECT coil was controlled to an effective value of $1 \mathrm{~mA}$ using the LCR meter. The frequency of this $A C$ magnetic field excitation was set to $50 \mathrm{kHz}$. The $A C$ magnetic field contribution was maintained lower than a quarter of the coercivity to ensure reversible AC magnetization changes, i.e., to avoid irreversible domain wall jumps, as recommended by the literature [40]. The resulting data provided by the LCR meter consisted of the sensor coil impedance modulus, phase, real and imaginary parts. A calibration procedure was carried out to take into account the parasitic impedances due to the electrical wires and contacts, and finally, the D\&D analytical expression was used to convert the impedance measurements into permeabilities. All the data were recorded using the Labiew ${ }^{\circledR}$ controller.

In parallel to this first configuration, a second one was developed to obtain a $\left|\underline{\mu_{I P}}\right|\left(H_{\text {surf }}\right)$ curve entirely discarded of dynamical effects (Fig. $6-$ b). In this second configuration, the B-coil was removed, the power amplifier and the frequency generator were replaced by a regulated power supply. The excitation coil current was controlled manually and measured through the shunt resistor. The MIP butterfly loop was obtained through discrete $\Delta H$ steps. The impedance measurement was done after a minimum of 50 seconds to avoid any transient phase issue. 

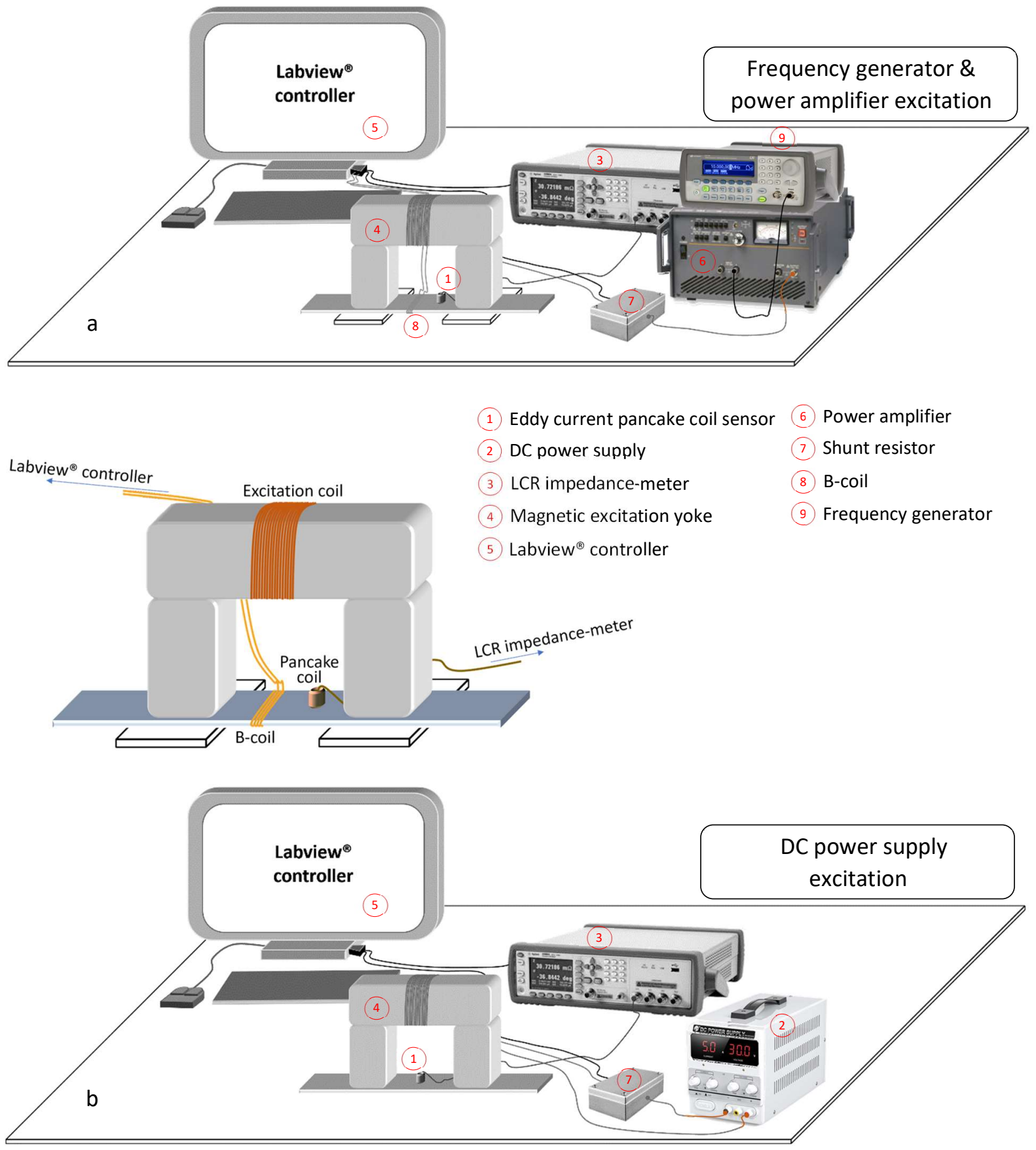

Fig. 6 - a Experimental set-up, first configuration for the simultaneous $\left|\underline{\mu_{I P}}\right|\left(H_{\text {surf }}\right)$ and $B_{a}\left(H_{\text {surf }}\right)$ characterization, Fig. 6 - b Experimental set-up, second configuration for the static $\left|\underline{\mu_{I P}}\right|\left(H_{\text {surf }}\right)$ characterization. 


\section{2-c) Tested specimen}

The tested specimens were all coming from the same batch. They were all electric steel, nonoriented grains (NO) FeSi laminations with a 3 wt \% silicon content and referenced 35JNE250. Tab. 1 below gives the physical properties as provided by the manufacturer.

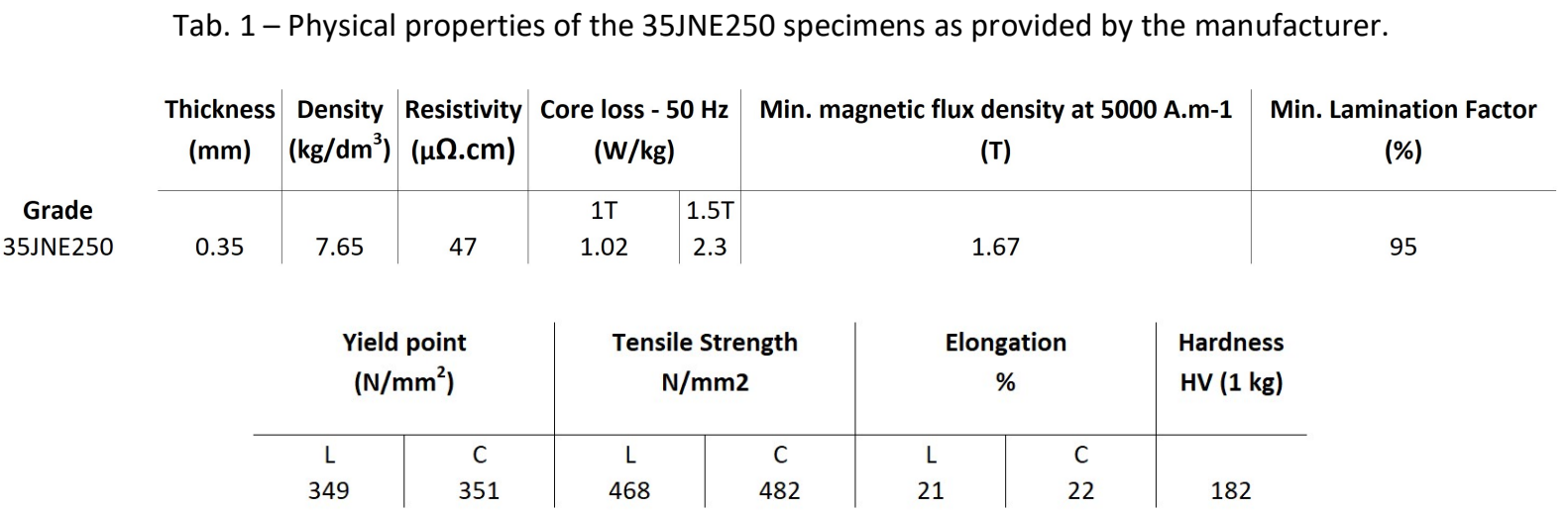

" $\mathrm{L}$ " and " $\mathrm{C}$ " means the specimens cut parallel and traverse to the rolling direction, respectively.

\section{3 - Experimental results}

Fig. 7 below depicted the $|\underline{Z}|(H)$ MIP butterfly loops measured with the Fig. 6 - a experimental set-up. $H$ had a sinusoidal shape, and tests were done from $1 \mathrm{mHz}$ to $1 \mathrm{~Hz}$. The frequency effect manifests itself by a shift of the coercivity (Fig. 7), the position of the peak read on the $H_{\text {surf }}$ axis and by an enlargement of the butterfly curve area and of the related indicators (curve width at $25 \%$ of $\mu_{\mathrm{MAX}}: \mathrm{DH} 25 \mu, 50 \%$ of $\mu_{\mathrm{MAX}}$ : $\mathrm{DH} 50 \mu$ and $75 \%$ of $\mu_{\mathrm{MAX}}: \mathrm{DH} 75 \mu$ as observed classically by the MIP NDT users [41]). 


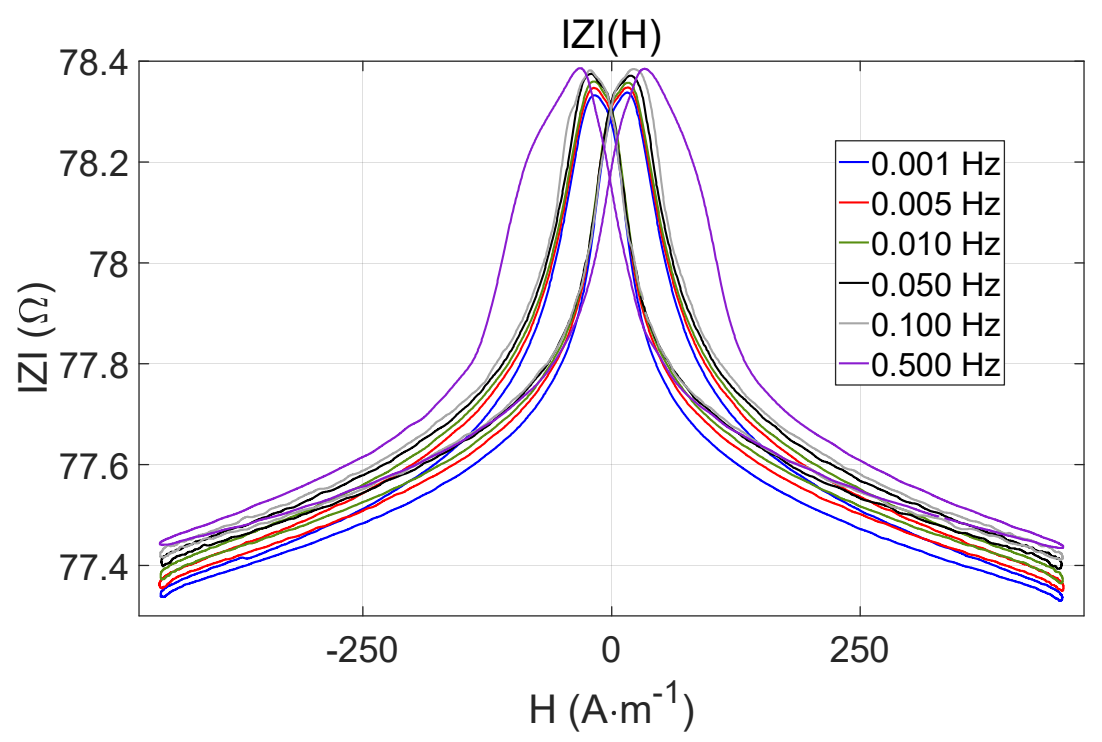

Fig. $7|\underline{Z}|(H)$ experimental loops with $H_{D C}$ frequency varying from $1 \mathrm{mHz}$ to $500 \mathrm{mHz}$.

Fig. 8 shows the related $\mu_{r_{I P}}(H)$ butterfly loops obtained once the interpolation process described in subsection 2-a is over. As envisaged, $|\underline{Z}|(H)$ and $\mu_{r_{I P}}(H)$ butterfly loops exhibit very similar frequency dependences.

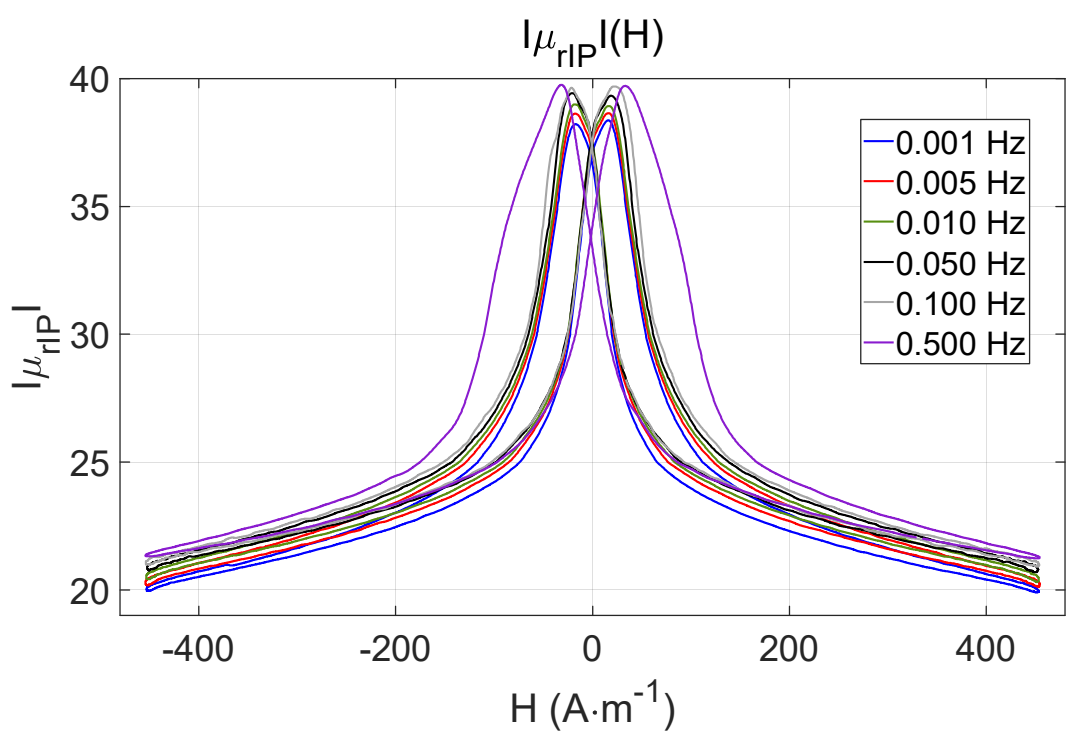

Fig. $8 \mu_{r_{I P}}(H)$ experimental loops with $H_{D C}$ frequency varying from $1 \mathrm{mHz}$ to $500 \mathrm{mHz}$. 
In the following figure (Fig. $9-\mathrm{a})$, the $B_{a}\left(H_{\text {surf }}\right)$ hysteresis cycles are plotted from $100 \mathrm{mHz}$ to $1 \mathrm{~Hz}$. The frequency effect clearly manifests itself by an enlargement of the hysteresis loop area and by an increase of the coercivity. Such observations are confirmed by the quasi constant increasing slope of Fig. $9-\mathrm{b}$ and $9-\mathrm{c}$, where the hysteresis loss $W$ and the coercivities $H_{c}$ are plotted as a function of the frequency $f$. It is worth noting that the hysteresis loss measurements (Fig. $9-$ c) are higher than the manufacturer observations $\left(350 \mathrm{~J}^{-3} \mathrm{~m}^{-3}\right.$ at $1.5 \mathrm{~T} 50 \mathrm{~Hz}$ ). These discrepancies are mostly due to the difference between the magnetic excitation status ( $B$ imposed vs $H$ imposed). Many additional sources of errors can justify such divergence, including the temperature, pressure, or deviating behaviors between specimen coming from different batches.

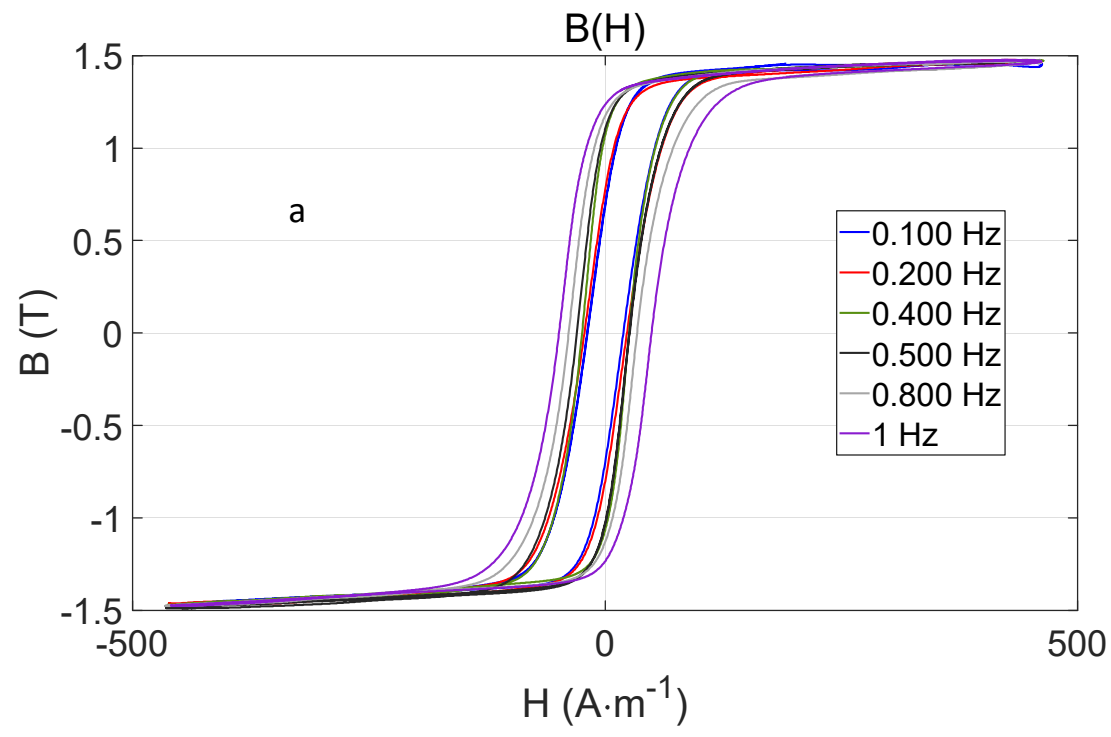



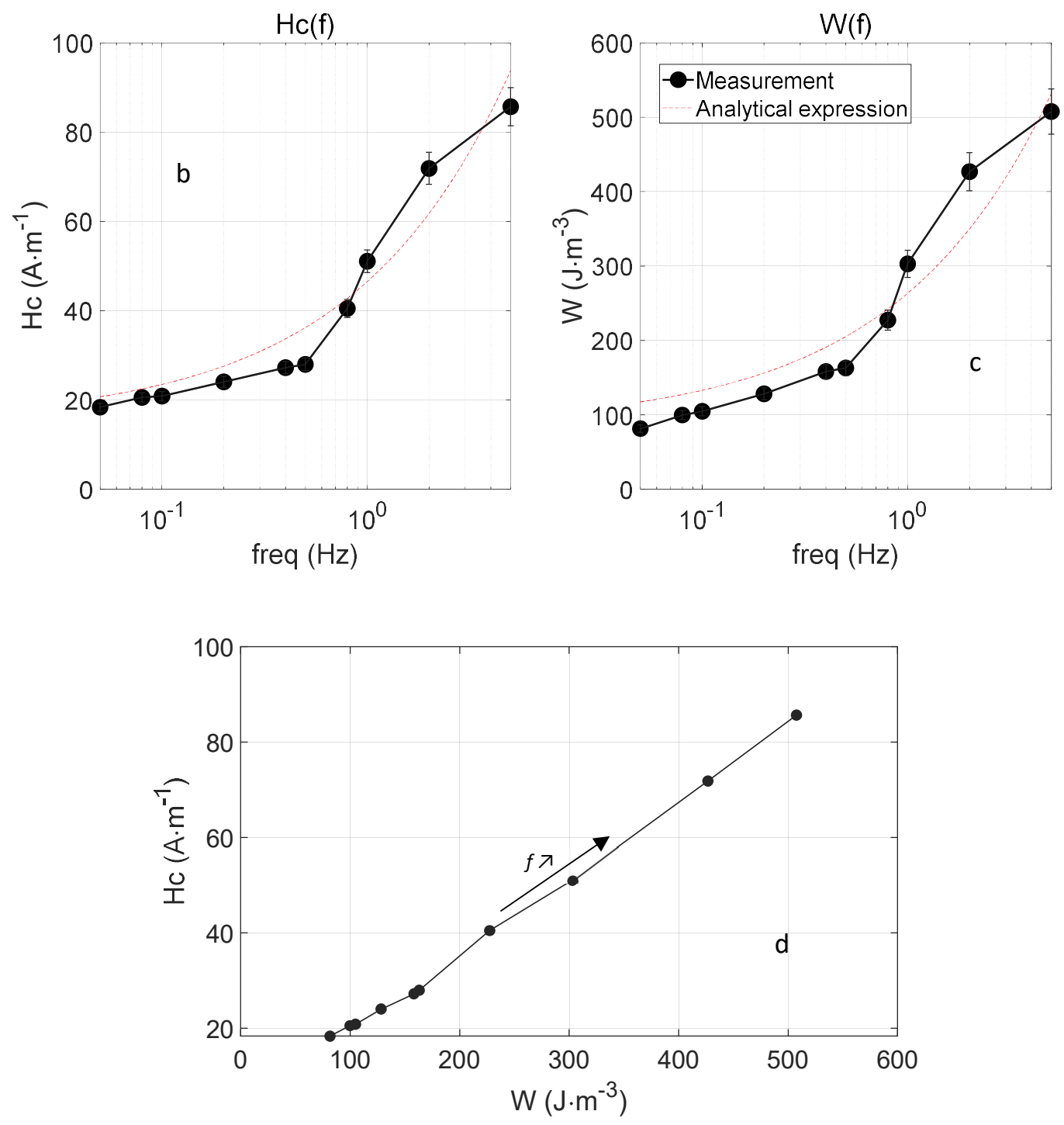

Fig. 9 - a $B(H)$ experimental loops with $H$ frequency varying from $100 \mathrm{mHz}$ to $1 \mathrm{~Hz}$, Fig. 9 - b Related coercivities $H c(f)$, Fig. 9 - c Related hysteresis loss $W(f)$ (hysteresis loop area). Fig. 9 - d Coercivity as a function of the hysteresis loss $\mathrm{Hc}(W)$.

It is interesting to observe the very close variations of $H_{c}(f)$ and $W(f)$ (Fig. 9 b, c, d), the correlation coefficient calculated with Matlab ${ }^{\circledR}$ "corr" function is equal to 0.9988 . It confirms, as noticed in simulation (Fig. 3) that both indicators can be used to evaluate the frequency effect.

In the following figure, Fig. 10, the frequency dependence of $H_{c_{\mu D P}}(f)$ and $H_{c_{\mu I P}}(f)$ is compared. The coercivities observed from both the permeability vs frequency curves show very similar trends in the $[50 \mathrm{mHz}-1 \mathrm{~Hz}]$ range, where both indicators can be observed correctly using 
both the experimental set-ups. This observation confirms our expectations and the parallel between the frequency dependence of the MIP and the MDP. It also ensures that the very lowfrequency behavior can be observed using the MIP butterfly loop.

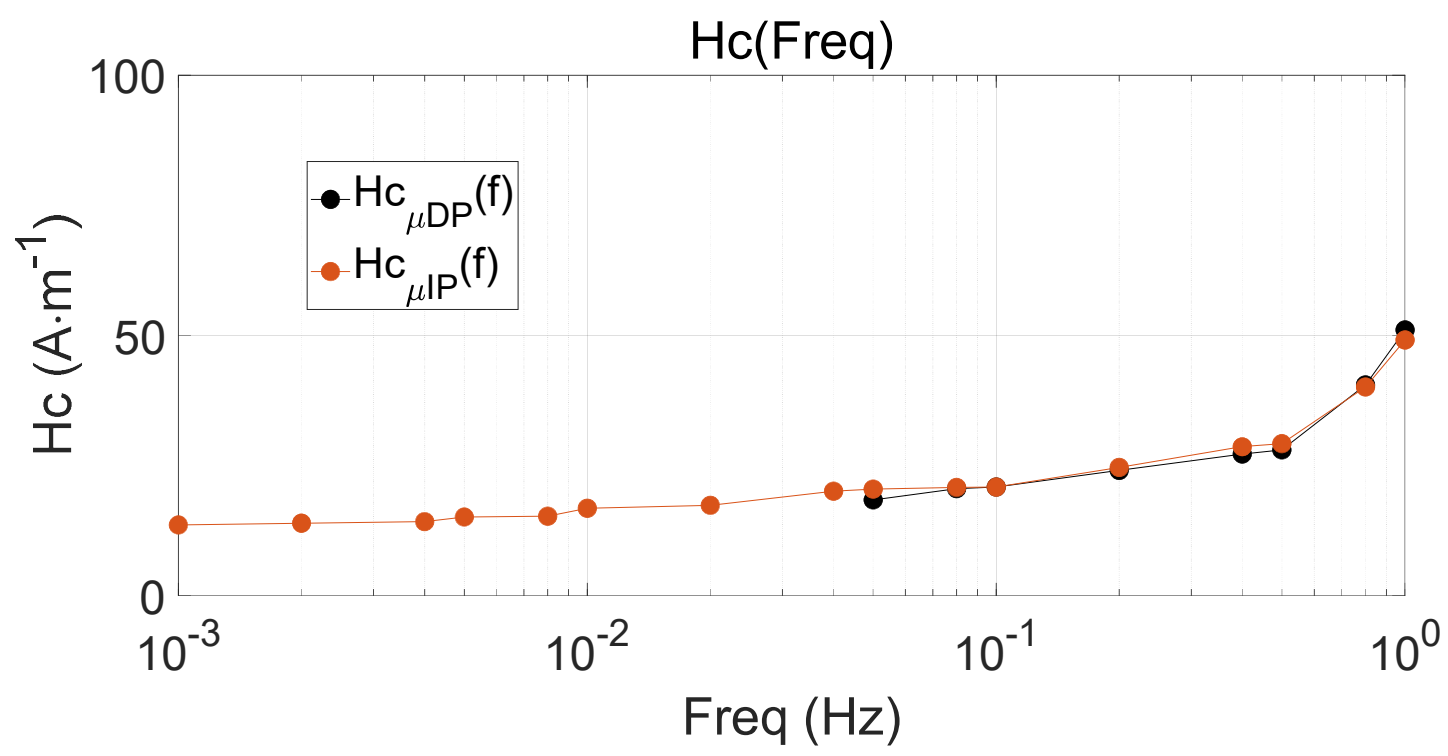

Fig. 10 Frequency dependence of the MDP and of the MIP coercivity.

The following result (Fig. 11) shows the MIP butterfly loops (| $\left.\underline{Z} \mid(H), \mu_{r_{I P}}(H)\right)$ obtained under full static conditions (i.e., measured with Fig. 2 - b experimental set-up). The static coercivity read on this curve is slightly larger than $12 \mathrm{~A} \cdot \mathrm{m}^{-1}$.
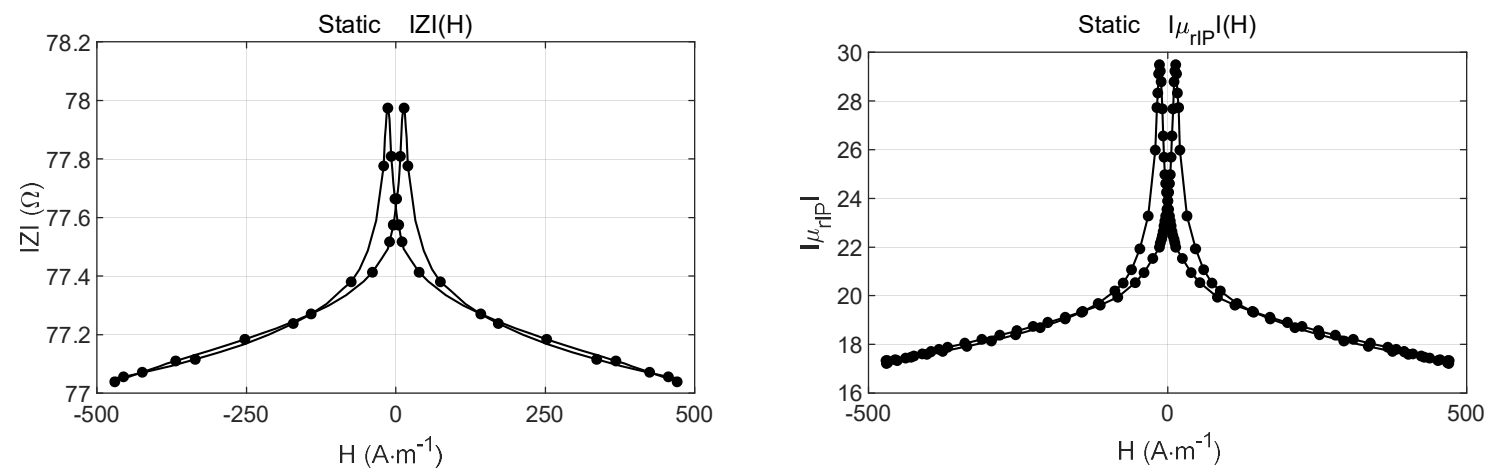

Fig. 11 Static $|\underline{Z}|(H), \mu_{r_{I P}}(H)$ butterfly loops as measured using Fig. 6 - b experimental set-up. 
The difference in terms of amplitude between Fig. 7, Fig. 8 at $f=0.001 \mathrm{~Hz}$ and Fig. $11-\mathrm{a}, \mathrm{b}$ can be explained by the transient phase observable on the evolution of $|\underline{Z}|$ for each $H_{D C}$ step and illustrated in Fig. 12 below:
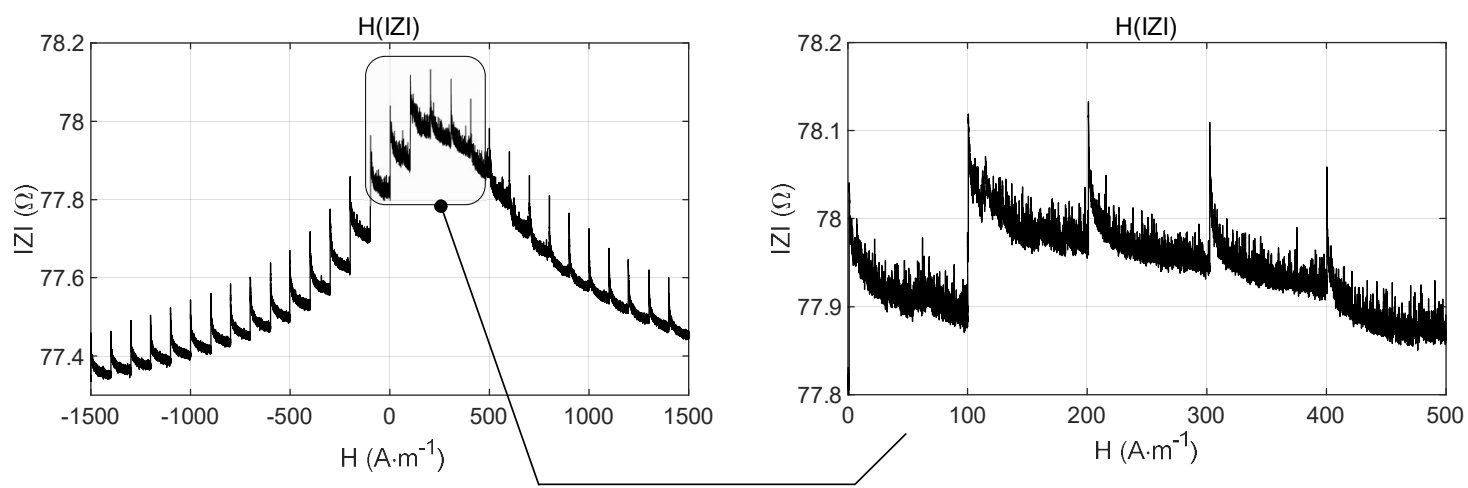

Fig. 12 Transient phase observation under increasing $H_{D C}$ steps.

In the experimental set-up description, we mentioned a wait of 50 seconds after every $H_{D C}$ field variation and before any measurement to ensure a complete end of this transient phase. Under sinus excitation and even in the low-frequency range, we can consider the magnetic material as in a permanently transient regime, high enough to generate a small difference with the real static curve. It is interesting to observe in Fig. 12, the additional high-frequency contribution close to the coercivity probably due to some Barkhausen noise activity.

By superimposing the coercivity read on the static MIP curve to the results show in Fig. 10 as illustrated below (Fig. 13), the quasi-static threshold can precisely be estimated. In the case of the $35 \mathrm{JNE} 250$ specimens tested in this work, this threshold is set equal to $4 \mathrm{mHz}$.
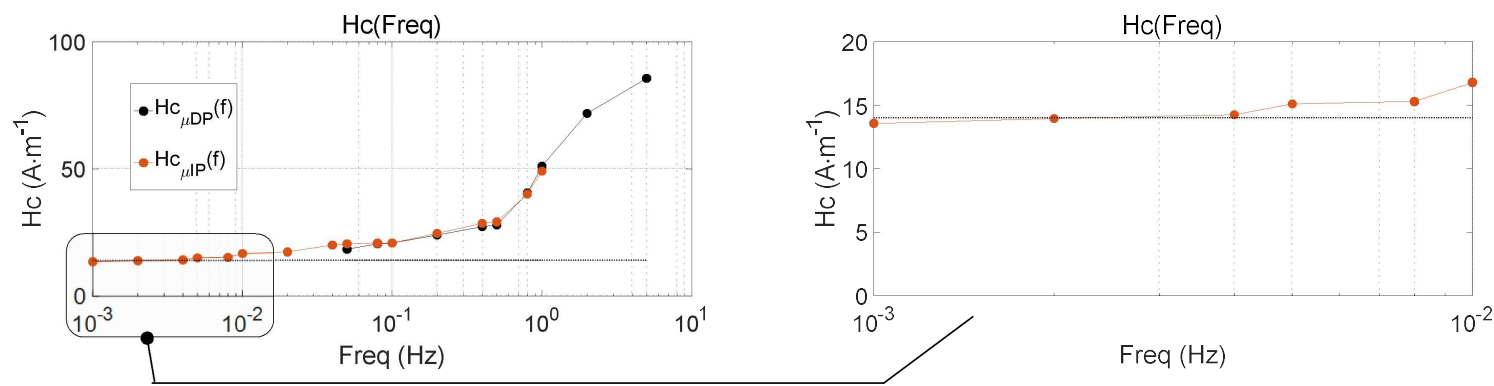
Fig. 13 Coercivities superimposition for the extraction of the quasi-static threshold.

Now, assuming the hysteresis losses as a function of the frequency curve $W(f)$ as following the exact same trajectory as the coercivity read on the $H_{c_{\mu I P}}(f)$ curves (Fig. 13), the lowfrequency range dependence of the magnetic losses can be estimated precisely through an analytical function (Eq. 14 and 15 below):

$$
\begin{gathered}
f \in[0.001-0.1] H z \\
E c(f)=E c_{o}\left(1+2.1 f^{0.57}\right) \\
W(f)=W_{0}\left(1+2.1 f^{0.57}\right)
\end{gathered}
$$

Where $E c_{o}$ and $W_{o}$ are the static values of the coercivity and hysteresis losses, respectively.

\section{4 - Conclusion and discussion}

The dynamic of the magnetization processes is complex. It relies on multiple interacting phenomena happening at different spatial scales and of different time constants. The very-lowfrequency magnetic behaviors (weakly above the quasi-static threshold) are rarely observed and evaluated. The main reason for this comes from the weak amplitude of the electromotive forces induced in the B-coil sensors of the classic characterization set-ups. In this work, we propose to address the low-frequency behavior of a nonoriented grain electric steel lamination by using magnetic incremental permeability experimental observations. In the first step of this work, we aimed at establishing the parallel and the similarities of the $\mathrm{B}(\mathrm{H})$, the $\left|\underline{\mu_{D P}}\right|(H)$ and the $\left|\underline{\mu_{I P}}\right|(H)$ frequency dependences in the limited low-frequency range where all the properties can be observed correctly. The very-low-frequency dependence of the $\left|\underline{\mu_{I P}}\right|(H)$ is proposed next, and a comparison between the real static result obtained under constant $H_{D C}$ bias is used to set the 
quasi-static threshold precisely. In the case of the 35JNE250 tested in this study, this frequency threshold is equal to $4 \mathrm{mHz}$. It is also interesting to analyze the scaling relation (Eq. 15) which has been proposed for the frequency of $W$ in the low-frequency range, especially the power factor equal to 0.57 , confirming a balance between the classic and the excess losses. 


\section{References}

[1] R.H. Pry, C.P. Bean, "Calculation of the energy loss in magnetic sheet materials using a domain model”, J. Appl. Phys., vol. 29, pp. 532-533, 1958.

[2] C.P. Bean, D.S. Rodbell, "Kinetics of magnetization in some square loop magnetic tapes", J. Appl. Phys, vol. 26, pp. 124-125, 1954.

[3] C.S. Schneider, S.D. Gedney, N. Ojeda-Ayala, M.A. Travers, "Dynamic exponential model of ferromagnetic hysteresis", Phys. B: Cond. Mat., vol. 607, 412802, 2021.

[4] H. Zhao, H.H. Eldeeb, Y. Zhang, Y. Zhang, G. Xu, O.A. Mohammed, "An improved core loss model of ferromagnetic materials considering High-frequency and non-sinusoidal supply", 2020 IEEE Ind. App. Soc. Ann. Meet., 10 - 16 Oct. 2020, Detroit, MI, USA.

[5] A. Magni, A. Sola, O. de la Barrière, E. Ferrara, L. Martino, C. Ragusa, C. Appino and F. Fiorillo, "Domain structure and energy losses up to $10 \mathrm{kHz}$ in grain-oriented Fe-Si sheets", AIP Advances 11, 015220, 2021.

[6] R. Corcolle, X. Ren, L. Daniel, "Effective properties and eddy current losses of soft magnetic composites”, J. Appl. Phys., vol. 129, 015103, 2021.

[7] B. Ducharne, P. Tsafack, Y.A. Tene Deffo, B. Zhang, G. Sebald, "Anomalous fractional magnetic field diffusion through cross-section of a massive toroidal ferromagnetic core", Com. in Nonlin. Sci. and Num. Sim., vol. 92, 105450, 2021.

[8] F.J.G. Landgraf, C. Ragusa, D.L.R. Junior, M.B.S. Dias, O. de la Barrière, F. Mazaleyrat, F. Fiorillo, C. Appino, L. Martino, "Loss decomposition in plastically deformed and partially annealed steel sheets", J. of Mag. and Mag. Mat., vol. 502, 166452, pp. 212-221, 2020.

[9] A. Daem, P. Sergeant, L. Dupré, M. N. Ibrahim, "Modeling interlocking effects on core losses in electrical steel”, IEEJ Trans. Elect. And Elect. Eng., vol. 15, iss. 12, pp. 1836-1843, 2020.

[10] X. Zhao, R. Wang, Z. Du, L. Dong, L. Liu, "Study on dynamic hysteresis and loss properties of silicon steel sheet under hybrid harmonic and DC bias excitation", IEEE Access, vol. 8, pp. 187343187352, 2020.

[11] K. Komeza, M. Dems, J.P. Lecointe, "Investigation of high frequency loss in electrical machine lamination considering skin effect hysteresis", Int. J. of App. Electromag. and Mech., vol. 64, iss. 7, pp. $1-16,2020$.

[12] R. Valenzuela, H. Montiel, M.P. Gutiérrez, I. Betancourt, "Characterization of soft ferromagnetic materials by inductance spectroscopy and magnetoimpedance", J. of Mag. and Mag. Mat., vol. 294, iss. 2, pp. 239-244, 2005.

[13] G. Bertotti, Hysteresis in Magnetism. San Diego, CA: Academic, 1998 
[14] G. Bertotti, "General properties of power losses in soft ferromagnetic materials", IEEE Trans. Magn., vol. 24, no. 1, pp. 621-630, 1988.

[15] S.E. Zirka, Y.I. Moroz, P. Marketos, A.J. Moses, "Loss separation in nonoriented electrical steels", IEEE Trans. Magn., vol. 46, no. 2, pp. 286-289, 2010.

[16] A. Broddefalk, M. Lindenmo, "Dependence of the power losses of a nonoriented 3\% Si-steel on frequency and gaude", J. of Mag. and Mag. Mat., vol. 304, pp. 586-588, 2006.

[17] M. A. Raulet, B. Ducharne, J.P. Masson, G. Bayada, "The magnetic field diffusion equation including dynamic hysteresis: a linear formulation of the problem", IEEE Trans. Magn., vol. 40, $n^{\circ}$ 2, pp. 872-875, 2004.

[18] S.E. Zirka, Y.I. Moroz, P. Marketos, A.J. Moses, "Viscosity-based magnetodynamic model of soft magnetic materials", IEEE Trans. Magn., vol. 42, no. 9, pp. 2121-2132, 2006.

[19] M. Petrun, S. Steentjes, "Iron-loss and magnetization dynamics in nonoriented electrical steel: 1-D Excitations up to high frequencies", IEEE Access, vol. 8, pp. 4568-4593, 2020.

[20] F. Preisach, "Über die magnetische nachwirkung", Zeitschrift Phys., vol. 94, no. 5-6, pp. 277302, 1935.

[21] D. C. Jiles and D. L. Atherton, "Theory of ferromagnetic hysteresis", J. Appl. Phys., vol. 55, no. 6, pp. 2115-2120, 1984.

[22] B. Ducharne, G. Sebald, D. Guyomar, G. Litak, "fractional model of magnetic field penetration into a toroidal soft ferromagnetic sample", Int. J. Dyn. Control., vol. 6, no. 1, pp. 89-96, 2018.

[23] S. Zhang, B. Ducharne, T. Uchimoto, A. Kita, Y.A. Tene Deffo, "Simulation tool for the Eddy Current Magnetic Signature (EC-MS) non-destructive method", J. of Mag. and Mag. Mat., vol. 513, 167221, 2020.

[24] N. Sadowski, N.J. Batistela, J.P.A. Bastos, M. Lajoie-Mazenc, "An inverse Jiles-Atherton model to take into account hysteresis in time-stepping finite-element calculations", IEEE Trans. Magn., vol. 38, n², pp. 797-800, 2002.

[25] V. Rubezic, L. Lazovic, A. Jovanovic, "Parameter identification of Jiles-Atherton model using the chaotic optimization method", COMPEL, 11, 0496, 2017.

[26] R. Liu, L. Li, "Analytical prediction model of energy losses in soft magnetic materials over broadband frequency range", IEEE Trans. Pow. Elect., vol. 36, no.2, 2021.

[27] M. Sowa, L. Majka, "Ferromagnetic core coil hysteresis modeling using fractional derivatives", Nonlin. Dyn., vol. 101, pp. 772-793, 2020. 
[28] B. Zhang, B. Gupta, B. Ducharne, G. Sebald, T. Uchimoto, "Preisach's model extended with dynamic fractional derivation contribution", IEEE Trans. Magn., vol. 54 iss. 3, 2018.

[29] B. Zhang, B. Gupta, B. Ducharne, G. Sebald, T. Uchimoto, "Dynamic magnetic scalar hysteresis lump model, based on JilesAtherton quasi-static hysteresis model extended with dynamic fractional derivative contribution", IEEE Trans. Magn., vol. 54, Iss. 11, 2018.

[30] IEC 60404-2, "Magnetic materials - Part 2: Methods of measurement of the magnetic properties of electrical steel strip and sheet by means of an Epstein frame", International Electrotechnical Commission, June 2008.

[31] IEC 60404-3, "Magnetic materials - Part 3: Methods of measurement of the magnetic properties of electrical steel strip and sheet by means of a single sheet tester", International Electrotechnical Commission, April 2010.

[32] IEC 60404-6, "Magnetic materials - Part 6: Methods of measurement of the magnetic properties of magnetically soft metallic and powder materials at frequencies in the range $20 \mathrm{~Hz}$ to $200 \mathrm{kHz}$ by the use of ring specimens", International Electrotechnical Commission, June 2003.

[33] B. Koprivica, A. Milovanovic, M. Plazinic, "Standard methods of measurement of the magnetic properties of electrical steel stip an sheet", XI Int. SAUM conference on Systems, Automatic Control and Measurement, Nis, Serbia, 2012.

[34] C.V. Dodd, W.E. Deeds, "Analytical solutions to eddy current probe coil problems", J. App. Phys. 39, (6), pp. 2829-2838, 1968.

[35] T. P. Theodoulidis, E. E. Kriezis, "Eddy Current Canonical Problems (With Applications to Nondestructive Evaluation)", GA, Norcross: Tech Science Press, 2006.

[36] S. Zhang, B. Ducharne, S. Takeda, G. Sebald, T. Uchimoto, "Identification of the ferromagnetic hysteresis simulation parameters using classic non-destructive testing equipment", J. of Mag. and Mag. Mat., 167971, 2021.

[37] B. Gupta, B. Ducharne, G. Sebald, T. Uchimoto, T. Miyazaki, T. Takagi, “Physical interpretation of the microstructure for aged $12 \mathrm{Cr}-\mathrm{Mo}-\mathrm{V}-\mathrm{W}$ steel creep test samples based on simulation of magnetic incremental permeability”, vol. 486, 165250, 2019.

[38] B. Gupta, T. Uchimoto, B. Ducharne, G. Sebald, T. Miyazaki, T. Takagi, "Magnetic incremental permeability non-destructive evaluation of $12 \mathrm{Cr}-\mathrm{Mo}-\mathrm{W}$-V Steel creep test samples with varied ageing levels and thermal treatments", NDT \& E Int., vol. 104, pp. 42-50, 2019.

[39] T. Matsumoto, T. Uchimoto, T. Takagi, G. Dobmann, B. Ducharne, S. Oozono, H. Yuya, "Investigation of Electromagnetic Nondestructive Evaluation of Residual Strain in Low Carbon Steels Using the Eddy Current Magnetic Signature (EC-MS) Method", J. of Mag. and Mag. Mat., vol. 479, pp. 212-221, 2019. 
[40] A. Yashan, G. Dobmann, "Measurements and semi-analytical modeling of incremental permeability using eddy current coil in the presence of magnetic hysteresis", in: F. Kojima, T. Takagi, S.S. Udpa, J. Pávó (Eds.), Electromagnetic Nondestructive Evaluation (VI), IOS press, pp. 150-157, 2002.

[41] B. Wolter, Y. Gabi, C. Conrad, "Nondestructive testing with 3MA - An overview of principles and applications", Appl. Sci, 9(6), 1068, 2019. 